\title{
Medida de consistência do concreto pelo abatimento do tronco de cone automatizado: sua validação e potencialidades
}

\author{
Measurement of concrete consistency through \\ an automated slump test device: \\ their validation and potentialities
}

João Batista Pereira ${ }^{1}$, Geraldo de Freitas Maciel $^{2}$

\author{
${ }^{1}$ Universidade Estadual Paulista - UNESP, Campus de Ilha Solteira, Laboratório de Hidrologia e Hidrometria - LH2, \\ Avenida Brasil Sul, 56, CEP: 15385-000, Ilha Solteira, São Paulo, Brasil. \\ ${ }^{2}$ Universidade Estadual Paulista - UNESP, Campus de Ilha Solteira, Departamento de Engenharia Civil, Alameda Bahia, \\ 550, CEP: 15385-000, Ilha Solteira, São Paulo, Brasil \\ e-mail: joao.pereira@unesp.br, geraldo.f.maciel@unesp.br
}

\section{RESUMO}

A partir do desenvolvimento de um aparato mecânico capaz de realizar automaticamente a medida de consistência do concreto pelo tronco de cone (slump test), este trabalho avaliou a capacidade do equipamento em simular o ensaio preconizado por norma. Medidas de consistência utilizando o ensaio padrão (tronco de cone - slump test) e o equipamento desenvolvido foram efetuadas para diferentes composições de concretos convencionais. Os resultados comprovaram a capacidade do equipamento em realizar o slump test, tanto o processo de levantamento do cone quanto a leitura do abatimento (slump), além de possibilitar menores erros experimentais associados ao operador durante o ensaio. Foram também avaliadas as propriedades tensão limite de escoamento e viscosidade plástica dos concretos produzidos, para isso modelos empíricos estabelecidos a partir de medidas reométricas (slump test modificado por meio do reômetro BTREOM), foram aplicados, os quais forneceram resultados compatíveis com os relatados na literatura. Enfatiza-se, porém, a necessidade de uma medida precisa da consistência, de maneira a não comprometer as avaliações reológicas do material, haja vista a elevada dependência (sensibilidade) da tensão limite de escoamento e mais ainda da viscosidade plástica àquela medida. Ademais, o equipamento proposto neste trabalho permitiu uma caracterização mais completa do escoamento durante o ensaio, fornecendo o comportamento temporal da descida do material (uma medida indireta da viscosidade) até o completo abatimento.

Palavras-chave: Slump test. Concreto. Reologia. Tensão limite de escoamento. Viscosidade plástica.

\begin{abstract}
From the development of a mechanical device capable of performing the slump test of concrete, this work evaluated the equipment's ability to simulate the test recommended by norm. Slumps using the standard test and the equipment developed were measured for different compositions of conventional concrete. The results confirmed the equipment's capacity to perform the slump test both in the lifting the Abrams cone and the slump reading, besides to allowing for less experimental errors associated with the operator during the test. Yield stress and plastic viscosity properties of the concretes produced were also evaluated - for this, empirical models based on rheometric measures were used (modified slump test via BTREOM rheometer) verifying results compatible with those reported in the literature. It should be emphasizes to need for a precise measurement of the slump, in order not to compromise the rheological evaluations of the material, given the high dependence (sensibility) of yield stress and even more of the plastic viscosity to that measure. Furthermore, the slump test equipment allowed for a more complete flow characterization during the test, providing the descent temporal behavior of the material (as an indirect measure of the viscosity) until final slump.
\end{abstract}

Keywords: Slump test. Concrete. Rheology. Yield stress. Plastic viscosity. 


\section{INTRODUÇÃO}

Na indústria da construção civil, a qualidade final das estruturas que utilizam materiais cimentícios depende do controle de qualidade desses materiais, tanto no estado fresco (características de trabalhabilidade) quanto no estado endurecido (propriedades mecânicas) [1]. Entretanto, o controle tecnológico do concreto se dá, na maioria das vezes, no estado endurecido, geralmente por meio de ensaios de resistência à compressão.

Nesse contexto, deve-se considerar também o controle das propriedades do concreto fresco, pois elas são fundamentais ao processo de execução das estruturas e garantia das propriedades mecânicas do concreto no estado endurecido [2]. Em se tratando do controle tecnológico do concreto fresco é necessário analisar os diferentes parâmetros do material a fim de atingir a trabalhabilidade adequada para cada situação, neste caso, a consistência, coesão, segregação, exsudação e o ar incorporado à mistura [3].

No estado fresco, determina-se, comumente, a consistência pelo ensaio de abatimento de tronco de cone - também conhecido como slump test, conforme Figura 1 - e definido, no Brasil, pela NBR 16889:2020 [4]. Esse ensaio avalia a consistência do material, permitindo que se controle a uniformidade do concreto e garantindo quesitos de aceitabilidade [5].

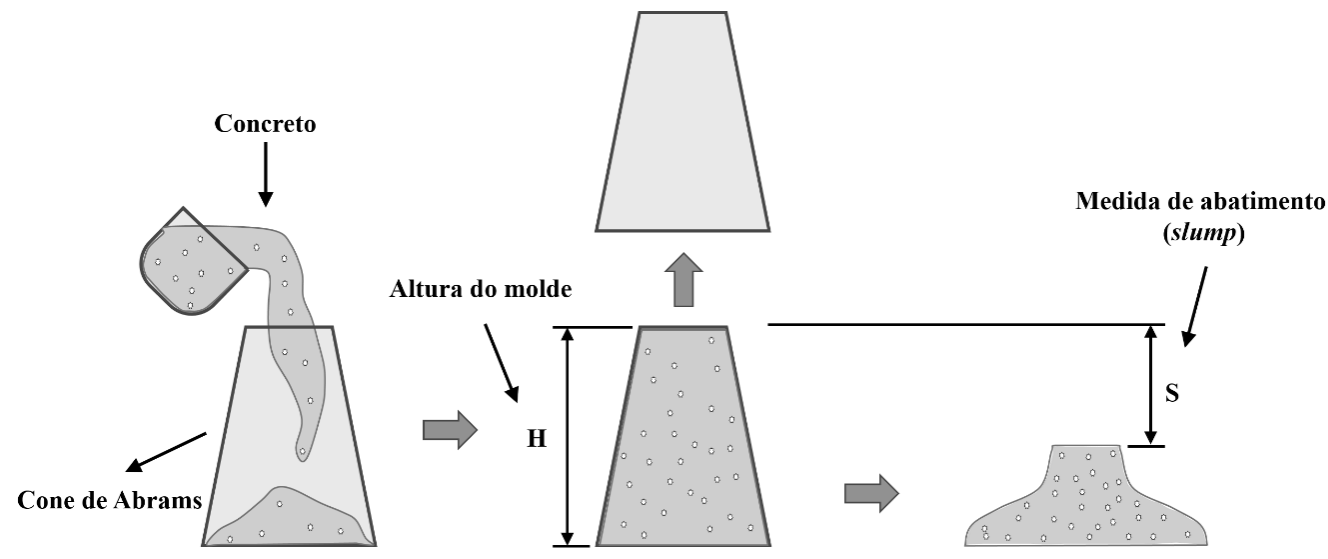

Figura 1: Ilustração do slump test.

O grande uso do slump test deve-se a sua simplicidade de execução e capacidade de detectar pequenas mudanças na composição da mistura como, por exemplo, variações no teor de água a partir da especificação original [3], além do baixo custo associado ao seu uso [6]. Mas é importante ressaltar que esse ensaio, em função do operador, pode fornecer respostas diferentes para uma mesma amostra [7], indicando forte dependência do operador.

Em função das mudanças dos processos construtivos e necessidades de novos materiais, a avaliação do comportamento de escoamento tem requerido mais rigor e uso de técnicas que permitam a medição dos níveis de tensão de cisalhamento e viscosidade dos materiais [8]. Nesse sentido, os materiais cimentícios no estado fresco podem ser convenientemente caracterizados por meio de medidas reológicas, a qual permite a avaliação de viscosidade, plasticidade e elasticidade desses materiais sob taxas de deformação ou cisalhamento [9].

Os materiais à base de cimento, notadamente os concretos e argamassas, são fluidos não-newtonianos, podendo exibir comumente dois comportamentos, o de fluido de BINGHAM e de HERSCHEL-BULKLEY $[10,11]$, representados, respectivamente, pelas Equações 1 e 2.

$$
\begin{gathered}
\tau=\tau_{0}+\mu_{\mathrm{p}} \dot{\gamma} \\
\tau=\tau_{0}+\mathrm{K}_{\mathrm{n}} \dot{\gamma}^{\mathrm{n}}
\end{gathered}
$$

sendo $\tau$ a tensão de cisalhamento, $\tau_{0}$ a tensão limite de escoamento, $\mu_{\mathrm{p}}$ a viscosidade plástica, $\dot{\gamma}$ a taxa de cisalhamento, $\mathrm{K}_{\mathrm{n}} \mathrm{o}$ índice de consistência e $\mathrm{n}$ o índice de escoamento. 
A análise reológica dos materiais requer o uso de ferramentas "especiais" (de maior precisão), como reômetros que apresentam elevado custo de aquisição [8]. Por outro lado, mecanismos mais simples e de fácil manuseio, capazes de simular a real aplicação do material podem ser usados, como a técnica de PASHIAS et al. [12], a técnica de abatimento do tronco de cone modificado proposta por FERRARIS e de LARRARD [11] e o teste da calha [13].

Assim, deve ser destacada a relação entre o ensaio de abatimento do tronco de cone (slump test) e a análise reológica - a medida de slump, utilizada inicialmente para avaliar a consistência do concreto fresco, permite que um importante parâmetro reológico seja inferido, a tensão limite de escoamento [12, 14-17]. Em função disso, o slump test tem sido cada vez mais empregado para análise de materiais de outros setores, tais como da indústria mineradora, petrolífera e alimentação, por se caracterizar como uma técnica robusta, barata e rápida na obtenção da tensão limite de escoamento $[12,18]$.

Como a medida de abatimento é capaz de se relacionar à medida de tensão limite de escoamento, FERRARIS e de LARRARD [11] desenvolveram o ensaio de abatimento do tronco de cone modificado. Esse ensaio permite descrever o comportamento reológico do concreto em termos dos parâmetros tensão limite de escoamento (em função da medida de slump) e viscosidade plástica (baseado em uma taxa média de descida do material durante o ensaio).

As correlações empíricas (Equações 3, 4 e 5) desenvolvidas por FERRARIS e de LARRARD [11] foram definidas a partir da caracterização reológica, via reômetro BTRHEOM, de uma ampla faixa de consistência de concretos produzidos com e sem aditivo redutor de água de amplo alcance (HRWRA - High-RangeWater Reducer Admixtures), considerando o modelo modificado de Bingham (baseado na aplicação do método dos mínimos quadrados para minimizar desvios entre os modelos de Bingham e Herschel-Bulkley). Segundo YAHIA e KHAYAT [19] e FEYS et al. [20], modelos modificados de Bingham mostram-se adequados para avaliação de materiais com comportamento reológico não linear e apresenta elevada precisão na estimativa da tensão limite de escoamento.

$$
\begin{array}{lc}
\tau_{0}=\frac{\rho}{347}(300-S)+212 & S>100 \mathrm{~mm} \\
\mu_{\mathrm{p}}=1,08 \cdot 10^{-3}(\mathrm{~S}-175) \rho \mathrm{T} & 200<\mathrm{S}<260 \mathrm{~mm} \\
\mu_{\mathrm{p}}=25 \cdot 10^{-3} \rho \mathrm{T} & \mathrm{S}<200 \mathrm{~mm}
\end{array}
$$

sendo S o abatimento do concreto $(\mathrm{mm}), \rho$ a massa específica de cada composição de concreto $\left(\mathrm{kg} / \mathrm{m}^{3}\right)$ e T o tempo parcial de abatimento (s), tempo esse necessário para o material descer $100 \mathrm{~mm}$.

Nesse contexto, o objetivo deste trabalho é apresentar um dispositivo automatizado capaz de realizar o ensaio de abatimento do tronco de cone (slump test), desde o levantamento do cone até a leitura da medida de abatimento e verificar seu funcionamento. Para isso, foram avaliadas as medidas de abatimento de diferentes composições de concreto segundo o ensaio padrão e o dispositivo desenvolvido. Também foi realizada uma caracterização reológica dos concretos analisados que na indisponibilidade de um reômetro para concreto, os autores utilizaram o método do slump test modificado de FERRARIS e de LARRARD [11], método esse desenvolvido a partir de uma larga base de dados reométricos de concretos medidos experimentalmente (tensão limite de escoamento e viscosidade plástica, diretamente do BTREOM ${ }^{1}$ ). Como as correlações (Equações 1, 2 e 3) estabelecidas por FERRARIS e de LARRARD [11] partem de medidas manuais clássicas do slump (S) e de um tempo característico (tempo parcial de abatimento T), a partir de cronômetro, portanto medidas suscetíveis à interferência do operador (forma como se eleva o cone e o tempo com que o cone é levantado mais rápido ou mais lento que o valor determinado em norma pode alterar a dinâmica do escoamento e consequentemente a medida de abatimento [21, 22]), esse artigo não só vem aplicar as correlações de FERRA-

\footnotetext{
${ }^{1}$ Parâmetros reológicos medidos a partir de reômetro de concreto BTREOM [11] calibrado e de confiabilidade reconhecida.
} 
RIS e de LARRARD [11], como inferir sua sensibilidade, qual seja, utiliza-se como caso teste um concreto de traço semelhante do banco de dados daqueles autores, agora medindo o abatimento e o tempo parcial de abatimento $\mathrm{T}$ de forma automatizada.

\section{MATERIAIS E MÉTODOS}

\subsection{Materiais e dosagem dos concretos}

Os materiais utilizados na composição dos concretos produzidos nesta pesquisa foram: cimento Portland composto com escória granulada de alto forno e classe de resistência de $32 \mathrm{MPa}$ aos 28 dias (CP II E 32), com massa específica de $3,05 \mathrm{~g} / \mathrm{cm}^{3}$; brita tipo 1 de origem basáltica, com massa específica de $2,87 \mathrm{~g} / \mathrm{cm}^{3}$, e areia natural média, com massa específica de $2,62 \mathrm{~g} / \mathrm{cm}^{3}$. O módulo de finura e a dimensão máxima desses agregados foram, respectivamente, 6,60 e $19 \mathrm{~mm}$ para a brita tipo 1 e 2,02 e 4,75 $\mathrm{mm}$ para a areia média (estando, segundo a NBR 7211:2009 [23], na zona utilizável inferior).

As composições de concretos utilizadas foram baseadas em concretos convencionais, sem uso de aditivo. A fim de obter uma ampla faixa de slump, três diferentes traços de concretos foram definidos, a saber: concretos T1, T2 e T3 com slumps, em mm, de $70 \pm 10,130 \pm 20$, e $180 \pm 30$, respectivamente. A Tabela 1 apresenta a composição de cada um dos traços produzidos.

Tabela 1: Composição dos traços de concreto produzidos.

\begin{tabular}{l|c|c|c}
\hline \multirow{2}{*}{ MATERIAIS } & \multicolumn{3}{|c}{ TRAÇO } \\
\cline { 2 - 4 } & T1 & T2 & T3 \\
\hline Água $\left(\mathrm{kg} / \mathrm{m}^{3}\right)$ & 213,20 & 221,15 & 233,00 \\
\hline Cimento $\left(\mathrm{kg} / \mathrm{m}^{3}\right)$ & 394,90 & 394,90 & 394,90 \\
\hline Brita tipo $1\left(\mathrm{~kg} / \mathrm{m}^{3}\right)$ & 994,00 & 994,00 & 994,00 \\
\hline Areia Média $\left(\mathrm{kg} / \mathrm{m}^{3}\right)$ & 803,00 & 803,00 & 803,00 \\
\hline Relação Água $/ \mathrm{Cimento}(\mathrm{a} / \mathrm{c})$ & 0,54 & 0,56 & 0,59 \\
\hline Massa específica $\left(\mathrm{kg} / \mathrm{m}^{3}\right)$ & 2406,00 & 2414,00 & 2426,00 \\
\hline
\end{tabular}

\subsection{Equipamento de abatimento do tronco de cone (slump test) automatizado}

O equipamento de abatimento do tronco de cone (slump test) automatizado, ilustrado na Figura 2, foi idealizado a fim de possibilitar um melhor controle de qualidade do concreto fresco por meio da redução de erros experimentais advindos do operador e, além disso, constituir-se em ferramenta de caracterização reológica de materiais por meio dos parâmetros medidos pelo dispositivo.

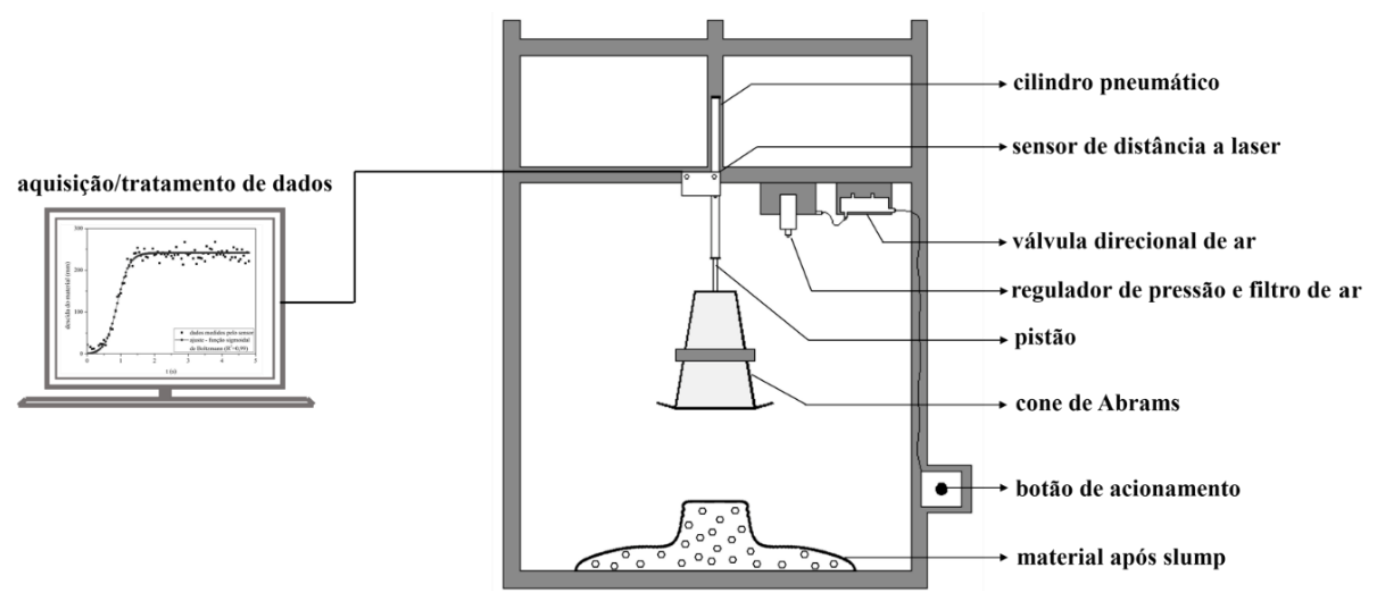

Figura 2: Ilustração esquemática do equipamento de abatimento do tronco de cone (slump test) automatizado. Ver link https://vimeo.com/446853491. 
O equipamento desenvolvido consiste de:

- Sistema pneumático para levantamento do tronco de cone (cone de Abrams). A partir desse sistema, é possível controlar a velocidade de levantamento do cone;

- Cone de Abrams com as dimensões definidas pela NBR 16889:2020 [4]. O processo de preenchimento do cone seguiu as definições da NBR 16889:2020 [4], preenchendo o molde segundo três (3) camadas iguais, sendo cada camada adensada com 25 golpes uniformemente distribuídos, de maneira manual, com uma haste de socamento;

- Sistema de leitura de abatimento, constituído por um sensor de medição de distância a laser, o qual é calibrado antes do início dos ensaios;

- Sistema de aquisição e tratamento de dados, constituído de placa de aquisição e computador.

Para a realização dos ensaios, definiu-se um tempo de $6 \pm 1 \mathrm{~s}$ para o levantamento do tronco de cone, de forma a respeitar o intervalo de 5 a 10 segundos recomendado pela NBR 16889:2020 [4].

\subsection{Medidas de abatimento}

Realizado o processo de mistura, foram determinadas as medidas de abatimento dos concretos T1, T2 e T3 por meio do ensaio de abatimento do tronco de cone e a partir do equipamento automatizado desenvolvido neste trabalho. Os dois métodos de ensaio foram realizados simultaneamente, sendo repetidos 8 (oito) vezes para cada traço.

A realização do ensaio de abatimento do tronco de cone se deu conforme os procedimentos definidos pela NBR 16889:2020 [4], já a obtenção do abatimento pelo equipamento automatizado também seguiu as recomendações descritas pela norma, entretanto o processo de levantamento do cone e de leitura de abatimento ocorreram a partir de mecanismos automatizados, sem interferência do operador.

Os dados obtidos a partir do equipamento automatizado permitiram, não apenas a definição do valor de abatimento dos concretos, mas também descrever o comportamento de escoamento dos concretos em função do tempo de ensaio (relação essa ligada ao comportamento de viscosidade do material [24, 25], ainda que trabalhos como o de CAMPOS et al. [26], avaliando argamassas, não tenha verificado correlação consistente entre tempo de escoamento e viscosidade plástica). Os dados obtidos por meio do equipamento desenvolvido são ilustrados pela Figura 3; para ajuste dos dados experimentais foi utilizada a função sigmoide de Boltzman (Figura 3.a), a qual apresentou elevada aderência aos dados coletados. Também foi medido o tempo T para o concreto se deslocar $100 \mathrm{~mm}$ (Figura 3.b) a fim de realizar a análise de viscosidade via modelo de FERRARIS e de LARRARD [11].

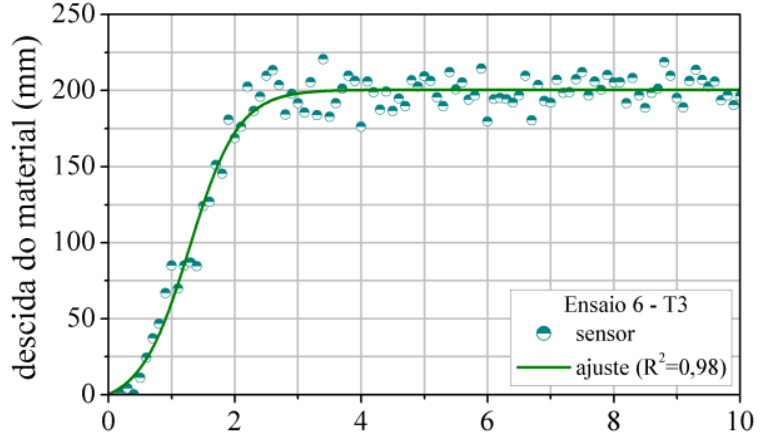

(a)

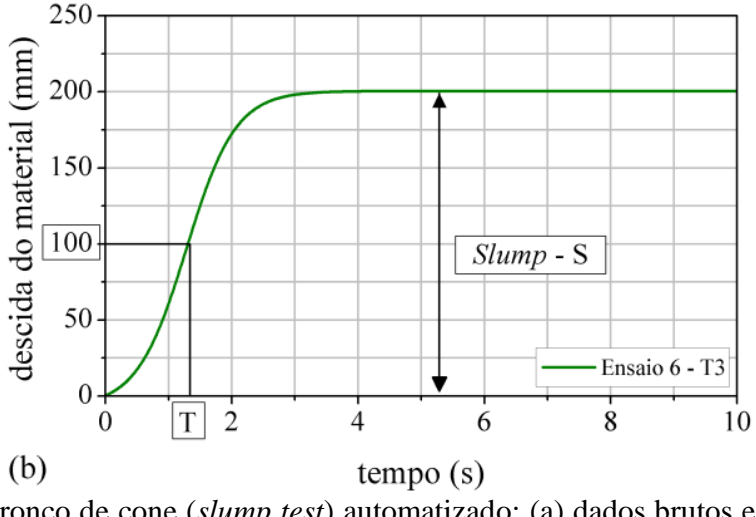

(b)

Figura 3: Dados obtidos no equipamento de abatimento do tronco de cone (slump test) automatizado: (a) dados brutos e ajuste empregado e (b) medida de abatimento e tempo de abatimento empregados nos modelos de FERRARIS e de LARRARD [11].

\section{RESULTADOS E DISCUSSÕES}

Os resultados de abatimento, em termos de valores médios e parâmetros estatísticos, medidos a partir do equipamento desenvolvido e aqueles obtidos pelo ensaio (padrão) de abatimento do tronco de cone normatizado são apresentados na Tabela 2. A Tabela 4 (vide Apêndice A), agrupa todos os dados experimentais medidos.

A partir dos dados dispostos na Tabela 2 e Figura 4, observa-se que os valores de abatimento obtidos no 
equipamento automatizado apresentaram, em todos os concretos analisados (T1, T2 e T3), a mesma ordem de grandeza daqueles obtidos pelo ensaio definido em norma, e baixos erros experimentais entre as duas formas de medição (erro máximo de $10 \%$, tendo o ensaio definido em norma como referência). Tal constatação demonstra a boa capacidade do equipamento automatizado em realizar o ensaio de abatimento do tronco de cone (slump test) e a leitura de abatimento.

Observa-se também que todos os ensaios, tanto padrão quanto automatizado, apresentaram baixo desvio padrão. A pequena variação dos valores de abatimento obtidos a partir do equipamento automatizado (desvio padrão máximo de $11 \mathrm{~mm}$ aproximadamente) atesta sua mínima interferência na realização do ensaio de abatimento do tronco de cone (slump test).

Tabela 2: Valores médios de abatimentos medidos pelo ensaio padrão e equipamento automatizado

\begin{tabular}{|c|c|c|c|c|c|c|}
\hline \multirow{2}{*}{$\begin{array}{l}\text { PARÂMETROS } \\
\text { ESTATÍSTICOS }\end{array}$} & \multicolumn{2}{|c|}{ CONCRETO T1 } & \multicolumn{2}{|c|}{ CONCRETO T2 } & \multicolumn{2}{|c|}{ CONCRETO T3 } \\
\hline & $\begin{array}{l}\text { Ensaio } \\
\text { padrão }\end{array}$ & $\begin{array}{c}\text { Ensaio } \\
\text { Automatizado }\end{array}$ & $\begin{array}{l}\text { Ensaio } \\
\text { padrão }\end{array}$ & \begin{tabular}{c|} 
Ensaio \\
automatizado
\end{tabular} & $\begin{array}{l}\text { Ensaio } \\
\text { padrão }\end{array}$ & $\begin{array}{c}\text { Ensaio } \\
\text { automatizado }\end{array}$ \\
\hline Slump médio (mm) & 73,80 & 66,30 & 134,00 & 128,15 & 194,70 & 189,65 \\
\hline Desvio padrão (mm) & 5,35 & 4,70 & 12,80 & 11,30 & 9,50 & 8,90 \\
\hline Slump máximo $(\mathrm{mm})$ & 79,00 & 73,40 & 149,50 & 139,50 & 206,70 & 202,20 \\
\hline Slump mínimo (mm) & 64,00 & 60,60 & 119,00 & 111,00 & 180,50 & 176,70 \\
\hline Coeficiente de variação (\%) & 7,25 & 7,15 & 9,10 & 8,80 & 4,90 & 4,70 \\
\hline Erro $(\%)$ & \multicolumn{2}{|r|}{10,10} & \multicolumn{2}{|r|}{4,30} & \multicolumn{2}{|r|}{2,60} \\
\hline
\end{tabular}

Com relação ao pequeno desvio padrão das medidas de abatimento a partir do ensaio padrão, pode-se dizer que a presença de um operador com conhecimento sobre as recomendações da norma NBR 16889:2020 [4] e que as seguiu à risca, controlando a velocidade de levantamento e a verticalidade do cone, possibilitou a padronização dos ensaios, reduzindo, assim, as variações de medida de slump. Em contrapartida, na realização desse ensaio por um operador desatento ou desconhecedor das recomendações da norma ou por diferentes operadores para uma mesma amostra, a variação das medidas de abatimento poderiam ser maiores, como relatado por POPOVICS [7].

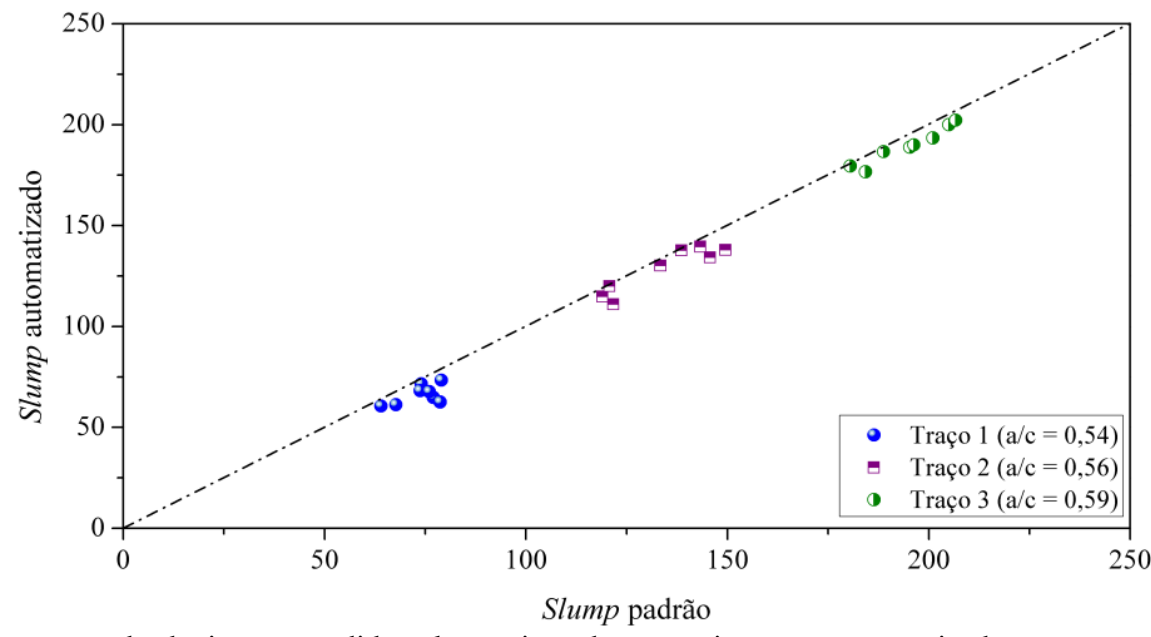

Figura 4: Comparação de abatimento medido pelo ensaio padrão e equipamento automatizado.

A Figura 5 ilustra o comportamento de escoamento dos três concretos analisados, bem como a faixa de erro (zona cinza) de cada um dos traços avaliados. Note que quanto maior a relação água cimento (a/c), maior a medida de abatimento, e consequentemente menor a medida de tensão limite de escoamento, conforme Figura 5 e 6.a, respectivamente. Essa constatação (relação abatimento e tensão limite de escoamento) pode ser verificada para diferentes materiais, desde materiais cimentícios e poliméricos, até lamosos [11, 15, 17, 27 e 28]. 

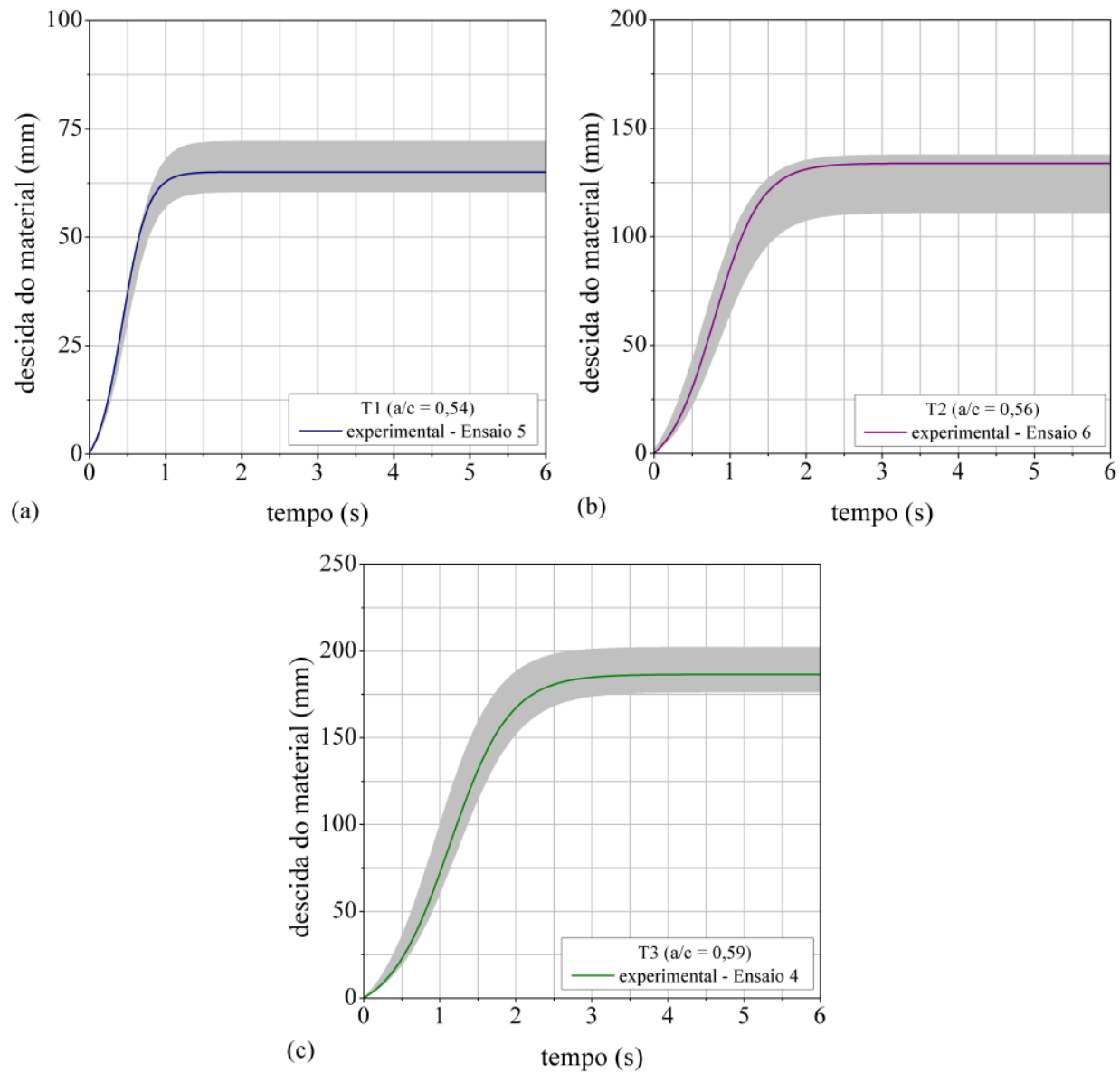

Figura 5: Comportamento de escoamento dos concretos analisados: (a) traço T1, (b) traço T2 e (c) traço T3.

Por meio dos dados obtidos no ensaio automatizado e da massa específica de cada composição de concreto, foram obtidos os parâmetros tensão limite de escoamento e viscosidade plástica dos concretos analisados, considerando as correlações estabelecidas por FERRARIS e de LARRARD [11], conforme Tabela 3.

Tabela 3: Valores médios dos parâmetros reológicos dos concretos produzidos a partir de medidas obtidas no equipamento desenvolvido. Os dados de todos os ensaios estão disponíveis na Tabela 4 do Apêndice A.

\begin{tabular}{c|c|c|c|c|c}
\hline CONCRETO & $\begin{array}{c}\text { MASSA ESPECÍFICA } \\
\left(\mathbf{k g} / \mathbf{m}^{3}\right)\end{array}$ & $\begin{array}{c}\text { SLUMP } \\
\mathbf{( m m )}\end{array}$ & $\begin{array}{c}\text { TEMPO PARCIAL DE } \\
\text { ABATIMENTO (s) }\end{array}$ & $\begin{array}{c}\text { TENSÃO LIMITE DE } \\
\text { ESCOAMENTO (Pa) }\end{array}$ & $\begin{array}{c}\text { V* } \\
\text { PLÁSCOSIDACA (Pa.s) }\end{array}$ \\
\hline $\mathrm{T} 1$ & 2406,00 & 66,30 & - & - & - \\
\hline $\mathrm{T} 2$ & 2414,00 & 128,20 & 1,55 & 1407,40 & 92,70 \\
\hline $\mathrm{T} 3$ & 2426,00 & 189,70 & 1,15 & 983,50 & 72,10 \\
\hline
\end{tabular}

* Para concretos com abatimento inferior a $100 \mathrm{~mm}$ não é possível medir o tempo parcial de abatimento (T) e calcular a viscosidade plástica; ** O modelo de previsão de tensão limite de escoamento vale para $\mathrm{S}>100$ $\mathrm{mm}$.

Note que no ensaio padrão não há a captura do tempo de deslocamento do material, sendo o abatimento a única medida fornecida pelo ensaio, conhecido como ensaio monoponto. Assim, o equipamento automatizado de medida de abatimento, além de reduzir os erros do operador, possibilita o monitoramento da evolução do abatimento do concreto com o tempo, o que permite a aplicação de modelos de previsão de viscosidade plástica e outras análises via correlações experimentais, bem como verificações de modelos analíticos e numéricos relacionados ao ensaio de abatimento do tronco de cone [29].

Ressalta-se que os valores dos parâmetros reológicos dos concretos observados nesta pesquisa (Figura 6) se enquadram nos valores experimentais obtidos por FERRARIS e de LARRARD [11], demonstrando que os concretos produzidos são compatíveis com as composições que validaram os modelos de correlação. FERRARIS e de LARRARD [11], considerando concretos sem HRWRA, obtiveram tensão limite de escoa- 
mento entre 700 e 1800 Pa e viscosidade plástica na faixa de 30 a 160 Pa.s, entretanto vale lembrar que o modelo por eles desenvolvido considera dados de concretos com e sem HRWRA.

Segundo BANFILL [30] o concreto apresenta tensão limite de escoamento na faixa de 500 a $2000 \mathrm{~Pa}$ e viscosidade plástica entre 50 e 100 Pa.s. Ainda que os concretos investigados na literatura, principalmente relacionados aos processos de bombeamento [31, 32], têm viscosidades inferiores a 120 Pa.s [33], há trabalhos que relatam valores superiores [34-36], como é o caso deste trabalho, em que a viscosidade superior a 120 Pa.s foi obtida, conforme pode se verificar na região I da Figura 6.b.

É necessário destacar que o modelo de FERRARIS e de LARRARD [11] foi desenvolvido a partir de caracterização reométrica via reômetro BTREOM, de forma que os resultados associados ao modelo de previsão estão intimamente relacionados ao reômetro utilizado. Nesse sentido, trabalhos técnicos realizados a partir da caracterização reológica de concretos por diferentes reômetros mostram a tendência do reômetro BTREOM em fornecer tensão limite de escoamento e viscosidade plástica superiores aos reômetros BML, CEMAGREF-IMG e TWO-POINT [37, 38]. Essa constatação justifica diferenças encontradas por outros autores na definição de modelos de correlação entre o abatimento do tronco de cone e propriedades reológicas de concretos como, por exemplo, o modelo de previsão de tensão limite de escoamento apresentado por WALLEVIK [39], o qual fez uso do reômetro BML.

A diferença entre modelos de previsão de tensão limite de escoamento a partir do abatimento de concretos é clara a partir do trabalho de WALLEVIK [39], no qual seu modelo empírico de previsão de tensão limite forneceu resultados inferiores aos definidos pelo modelo de FERRARIS e de LARRARD [11]. Essa constatação permite afirmar, conforme relatado por CHIDIAC et al. [40], que os níveis de correlação e desviospadrão de modelos de previsão de parâmetros reológicos podem ser distintos em função do uso de reômetros diferentes.
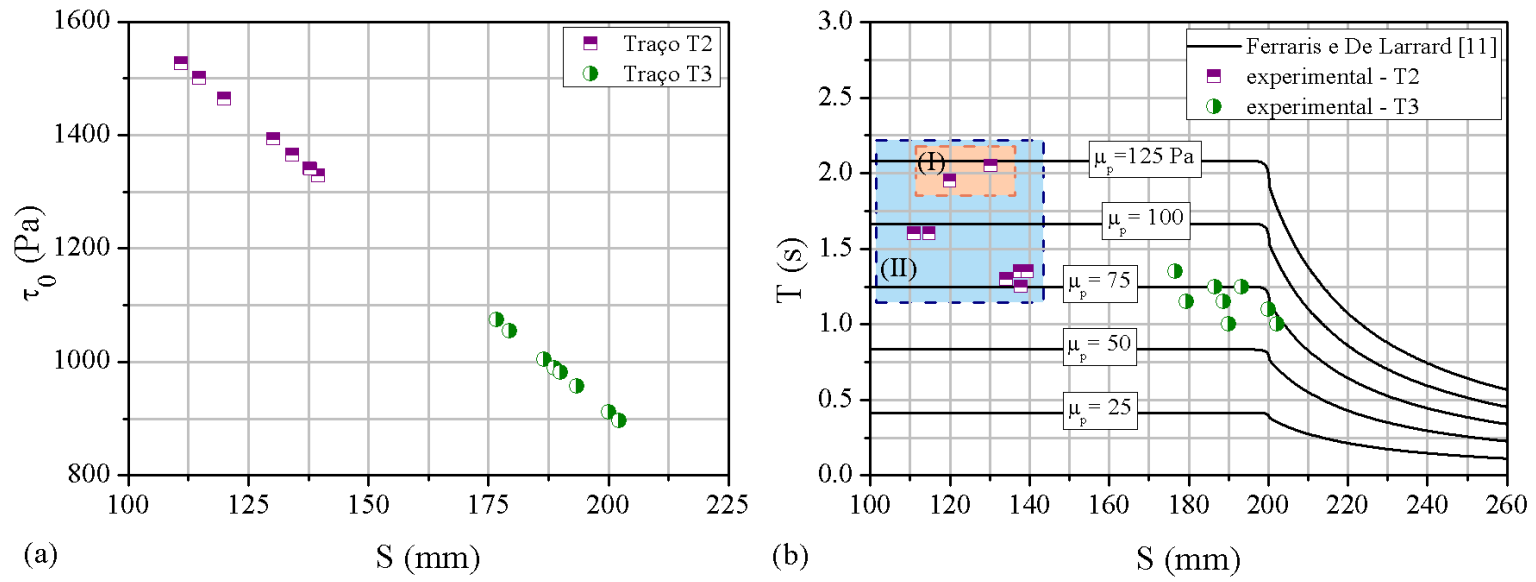

Figura 6: Parâmetros reológicos obtidos a partir das formulações de FERRARIS e de LARRARD [11]: (a) tensão limite de escoamento e (b) viscosidade plástica.

Os modelos de previsão de tensão limite de escoamento e viscosidade a partir do ensaio de abatimento do tronco de cone são fortemente dependentes da medida de abatimento, visto que a partir de varredura da literatura sobre modelos de previsão reológica via ensaio de abatimento ficou confirmada o quão dependente das medidas do abatimento são os modelos preditivos, sejam as correlações de base empíricas [41, 39], sejam aquelas definidas via simulação numérica $[42-44,16)$, e até mesmo as que partiram de desenvolvimento teórico [45- 47]. Isso também se aplica ao modelo de FERRARIS e de LARRARD [11] utilizado neste trabalho, como demonstrado a partir das derivadas das Equações 3 e 5, conforme resultados descritos pelas Equações 6 e 7:

$$
\left|\frac{\mathrm{d} \tau_{0}}{\mathrm{dS}}\right|=\frac{\rho}{347}=2,9 \cdot 10^{-3} \rho
$$$$
\mathrm{S}>100 \mathrm{~mm}
$$ 


$$
\left|\frac{\mathrm{d} \mu_{\mathrm{p}}}{\mathrm{dT}}\right|=25 \cdot 10^{-3} \rho \quad \mathrm{S}<200 \mathrm{~mm}
$$

Note que as derivadas obtidas são função da massa específica, parâmetro esse da ordem de $2 \times 10^{3}$, implicando em elevada sensibilidade da tensão limite de escoamento à medida de abatimento e da medida de viscosidade plástica (para $\mathrm{S}$ menor que $200 \mathrm{~mm}$ ) ao parâmetro tempo parcial de abatimento T. Comparando as Equações 6 e 7 verifica-se que a viscosidade plástica apresenta sensibilidade 10 vezes superior quando comparada ao parâmetro tensão limite de escoamento.

Considerando as massas específicas dos concretos deste trabalho, $\rho=2400 \mathrm{~kg} / \mathrm{m}^{3}$, é possível afirmar que a tensão limite de escoamento via modelo de FERRARIS e de LARRARD [11] sofre variações de 6,90 Pa para cada $1 \mathrm{~mm}$ de abatimento, justificando, mesmo com o rigor empregado na execução do ensaio de abatimento do tronco de cone (slump test) (equipamento automatizado e ensaio padrão), as diferenças de tensão limite de escoamento apresentadas na Figura 7. Tais erros são inerentes da correlação empregada, erro médio de 162 Pa segundo [11], entretanto podem ser reduzidas a partir da medida automatizada de abatimento (menor barra de erro, conforme Figura 7). Já a medida de viscosidade plástica, na faixa de $\mathrm{S}<200 \mathrm{~mm}$, é alterada em 6 Pa.s a cada variação de $0,1 \mathrm{~s}$ (ou ainda 60 Pa.s a cada $1 \mathrm{~s}$ ), o que implica em variações maiores caso haja faixa de tempo parcial de abatimento T elevado, como é observado na região II da Figura 6.b.

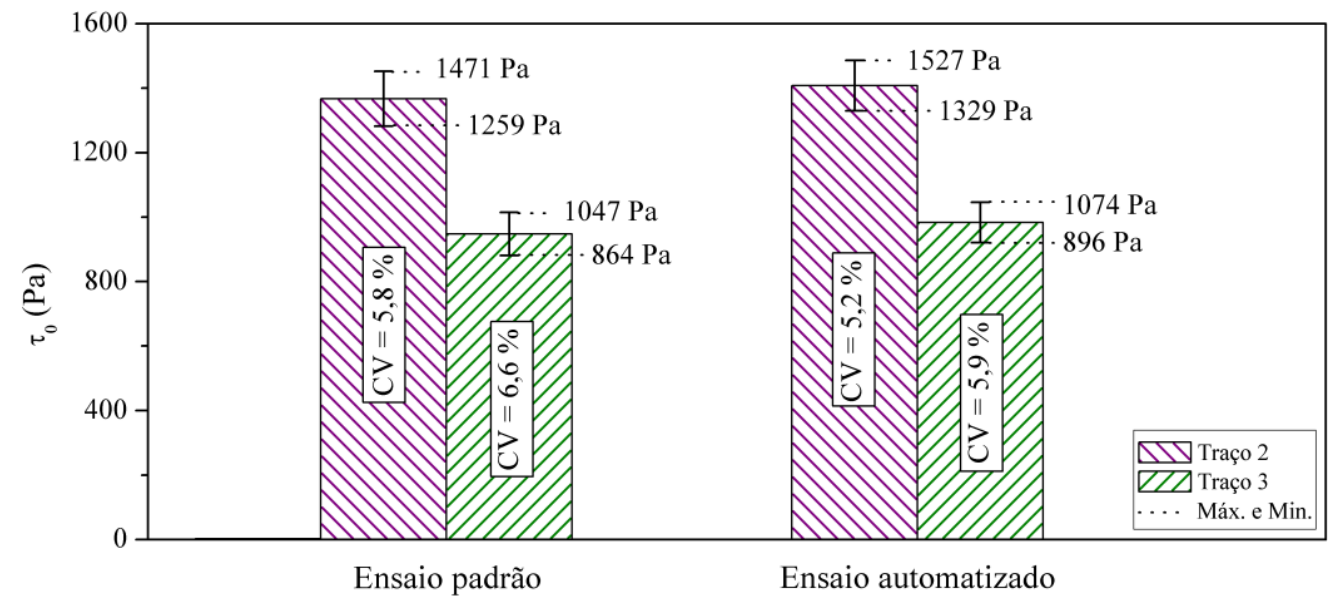

Figura 7: Parâmetro tensão limite de escoamento obtido a partir do modelo de FERRARIS e de LARRARD [11]. Valores médios, limites superior e inferior, e coeficiente de variação $(\mathrm{CV})$ das medidas de tensão limite de escoamento. Dados completos presentes na Tabela 4.

É importante notar que os modelos empíricos que utilizam o ensaio de abatimento do tronco de cone (slump test) contabilizam as incertezas referentes ao experimental, dessa forma tais modelos incorporam erros [48, 49]. Tais erros podem ser aumentados quando do uso de medidas advindas de ensaio de abatimento do tronco de cone (slump test) com baixo rigor de execução. Dessa forma, quanto maior o controle deste ensaio, como o promovido pelo equipamento automatizado, menores as incertezas sobre os parâmetros reológicos obtidos via correlação, seja no caso de materiais cimentícios de reologia Binghamiana, seja, sobretudo, para materiais mais ajustáveis a modelos reológicos mais complexos. Nesse sentido, Mahmoodzadeh e ChiDIAC [49], a partir de um dispositivo mecânico capaz de promover o levantamento do cone de ABRAMS [46] e elevar a precisão das informações coletadas, melhoraram os coeficientes de determinação $\left(R^{2}\right)$ de modelos baseados no abatimento de concretos, sobretudo o modelo de previsão de viscosidade plástica.

Ademais, o monitoramento da evolução temporal da descida do material, proporcionado pelo equipamento desenvolvido, descrito neste artigo, permite caracterizar o parâmetro temporal de abatimentos inferiores a $100 \mathrm{~mm}$, domínio esse não avaliado pelo ensaio de abatimento do tronco de cone (slump test) modificado e que inviabiliza a avaliação do progresso da viscosidade plástica de concretos ao longo do tempo [50].

\section{CONCLUSÕES}

A partir do estudo realizado, pôde-se verificar que o equipamento desenvolvido para a automatização do en- 
saio do abatimento do tronco de cone (slump test) resultou em valores de abatimentos próximos e de mesma ordem de grandeza daqueles medidos pelo ensaio definido pela NBR 16889:2020 [4], além de redução de erros associados ao ensaio (coeficiente de variação máximo inferior a 9\%), demonstrando, portanto, sua funcionalidade e operacionalidade para uma ampla faixa de abatimento.

O equipamento desenvolvido fornece dados que permitirão o desenvolvimento e uso de correlações matemáticas mais afinadas e capazes de obter os parâmetros reológicos, inclusive aqueles que dependam, por exemplo, da evolução temporal da descida do material (caso da viscosidade), e sem limitação da faixa de abatimento a ser avaliada.

Também se pode vislumbrar estender seu uso para diferentes materiais (de base cimentícia, mineralógica e até mesmo polimérica), diminuindo a ação do operador (ou operadores ou eventual operador inexperiente ou desconhecedor da norma) no ensaio, gerando, consequentemente, maior facilidade, confiabilidade e menor dispersão nos resultados de slump e, por conseguinte, na previsão de tensão limite de escoamento e viscosidade.

Por fim, é importante se atentar aos elevados erros apresentados pelos modelos empíricos utilizados neste trabalho, cujos erros médios de tensão limite de escoamento e viscosidade são respectivamente, de 162 Pa e 66 Pa.s. Isso evidencia a necessidade de atenção (no que diz respeito ao conhecimento e sensibilidade do método) no processo de obtenção e/ou uso de modelos empíricos de parâmetros reológicos, bem como no elevado rigor na execução e obtenção de dados durante o ensaio de abatimento do tronco de cone (slump test), no contexto de soluções alternativas em reologia, afora o uso de reômetros, que por si só exige também operador experiente e equipe com preparo para fazer as boas interpretações físicas de resultados não necessariamente todos comparáveis quando advindos, sobretudo, de reômetros distintos, manuseados e interpretados por operadores distintos.

\section{BIBLIOGRAFIA}

[1] KOVLER, K., ROUSSEL, N., "Properties of fresh and hardened concrete", Cement and Concrete Research, v. 41, n. 7, pp. 775-792, 2011.

[2] SURAHYO, A., Physical Properties of Concrete, In: Concrete Construction, Springer, pp. 61-88, 2019.

[3] MEHTA, P.K.; MONTEIRO, P.J.M., Concrete microstructure, properties and materials, McGraw-Hill, 2006.

[4] ASSOCIAÇÃO BRASILEIRA DE NORMAS TÉCNICAS, NBR 16889: Concreto: Determinação da consistência pelo abatimento do tronco de cone, Rio de Janeiro, 2020.

[5] BARTOS, P.J.M., et al., Workability and Rheology of fresh concrete: compendium of tests, Report of RILEM Technical Committee TC 145-WSM, Workability of Special Concrete Mixes, Cachan: RILEM, 2002.

[6] SAAK, A.W., et al., "A generalized approach for the determination of yield stress by slump and slump flow", Cement and Concrete Research, v. 34, n. 3, pp. 363-371, 2004.

[7] POPOVICS, S., Fundamentals of Portland Cement Concrete - a Quantitative Approach: Fresh concrete, John Wiley \& Sons, 1982.

[8] WALLEVIK, O.H., WALLEVIK, J.E., "Rheology as a tool in concrete science: The use of rheographs and workability boxes", Cement and Concrete Research, v. 41, n. 12, pp. 1279-1288, 2011.

[9] JIAO, D., et al., "Effect of constituents on rheological properties of fresh concrete - A review", Cement and Concrete Composites, v. 83, pp. 146-159, 2017.

[10] TATTERSALL, G.H., BANFILL, P.F.G., The rheology of fresh concrete, Pitman, 1983.

[11] FERRARIS, C. F., de LARRARD, F., Testing and modeling of fresh concrete rheology, In: Report NISTIR 6094, National Institute of Standards and Technology, 1998.

[12] PASHIAS, N., et al., "A fifty cent rheometer for yield stress measurement", Journal of Rheology, v. 40, n. 6, pp. 1179-1189, 1996.

[13] GAO, J., FOURIE, A., "Using the flume test for yield stress measurement of thickened tailings", Minerals Engineering, v. 81, pp. 116-127, 2015. 
[14] CHRISTENSEN, G., Modelling the flow of fresh concrete: the slump test, Tese de D.Sc., Faculty of Princeton University, Princeton University, Princeton, 1991.

[15] CLAYTON, S., et al., "Analysis of the slump test for on-site yield stress measurement of mineral suspensions", International Journal of Mineral Processing, v. 70, n. 1, pp. 3-21, 2003.

[16] ROUSSEL, N., "Correlation between yield stress and slump: comparison between numerical simulations and concrete rheometers results", Materials and Structures, v. 39, n. 4, pp. 501-509, 2006.

[17] PIERRE, A., et al., "Extension of spread-slump formulae for yield stress evaluation", Applied Rheology, v. 23 , n. 6, 2013.

[18] GARCIA-BERNET, D., et al., "Rapid measurement of the yield stress of anaerobically digested solid waste using slump tests", Waste Management, v. 31, n. 4, pp. 631-635, 2011.

[19] YAHIA, A., KHAYAT, K.H., "Analytical models for estimating yield stress of high-performance pseudoplastic grout", Cement and Concrete Research, v. 31, n. 5, pp. 731-738, 2001.

[20] FEYS, D., et al., "Extension of the Reiner-Riwlin equation to determine modified Bingham parameters measured in coaxial cylinders rheometers", Materials and Structures, v. 46, n. 1-2, pp. 289-311, 2013.

[21] LIU, Y., et al., "Axisymmetric viscoplastic dambreaks and the slump test", Journal of Non-Newtonian Fluid Mechanics, v. 258, pp. 45-57, 2018.

[22] GAO, J., FOURIE A., Spread is better: an investigation of the mini-slump test, Minerals Engineering, v. 71, pp. 120-132, 2015.

[23] ASSOCIAÇÃO BRASILEIRA DE NORMAS TÉCNICAS, NBR 7211: Agregados para concreto: Especificação, Rio de Janeiro, 2009.

[24] CHOI, M.S., et al., "Estimation of rheological properties of UHPC using mini slump test", Construction and Building Materials, v.106, pp. 632-639, 2016.

[25] TREGGER, N., et al., "Identifying viscosity of cement paste from mini-slump-flow test", ACI Materials Journal, v.105, n. 6, pp. 558, 2008.

[26] CAMPOS, R.S., et al., "Influência dos agregados reciclados nas propriedades reológicas e mecânicas do concreto autoadensável”, Matéria (Rio de Janeiro), v. 23, n. 1, 2018.

[27] MACIEL, G.F., et al., "Análise comparativa da tensão limite de escoamento de argamassas por meio da técnica de Pashias e reometria rotacional”, Matéria (Rio de Janeiro), v. 21, n. 4, pp. 866-879, 2016.

[28] LI, W., et al., "Analytical and experimental investigation of the relationship between spread and yield stress in the mini-cone test for cemented tailings backfill", Construction and Building Materials, v. 260, pp. 119770, 2020.

[29] SAO, Y.T., et al., "Validação numérica de um escoamento de fluido não-newtoniano no âmbito de corridas de lamas", In: XXVIII Congresso Latino-Americano de Hidráulica, Buenos Aires, Set. 2018.

[30] BANFILL, P.F.G., et al., In: FERRARIS, C. F., BROWER, L. E. (Eds.), Comparison of Concrete Rheometers: International Tests at LCPC (Nantes, France), In: Report NISTIR 6819, National Institute of Standard and Technology, 2001.

[31] SECRIERU, E., et al., "Changes in concrete properties during pumping and formation of lubricating material under pressure", Cement and Concrete Research, v. 108, pp. 129-139, 2018.

[32] CHOI, M.S., et al., "Effect of the coarse aggregate size on pipe flow of pumped concrete", Construction and Building Materials, v. 66, pp. 723-730, 2014.

[33] SOUALHI, H., et al., "New model to estimate plastic viscosity of eco-friendly and conventional concrete", Construction and Building Materials, v. 135, pp. 323-334, 2017.

[34] KIM, J., et al., "Concrete pumping prediction considering different measurement of the rheological properties", Construction and Building Materials, v. 171, pp. 493-503, 2018.

[35] MENDES, M., et al., "Avaliação dos parâmetros de autoadensabilidade e de reologia do concreto autoadensável”, Matéria (Rio de Janeiro), v. 22, n. 4, 2017.

[36] GHANBARI, A., KARIHALOO, B.L., "Prediction of the plastic viscosity of self-compacting steel fibre reinforced concrete", Cement and Concrete Research, v. 39, n. 12, pp. 1209-1216, 2009. 
[37] BANFILL, P.F.G., "The rheology of fresh cement and concrete-rheology review", British Society of Rheology, 61, v. 130, 2006.

[38] BEAUPRÉ, D., et al., In: FERRARIS, C. F., BROWER, L. E. (Eds.), Comparison of Concrete Rheometers: International Tests at MB (Cleveland, USA), In: Report NISTIR 7154, National Institute of Standard and Technology, 2004.

[39] WALLEVIK, J.E., "Relationship between the Bingham parameters and slump", Cement and Concrete Research, v. 36, n. 7, pp. 1214-1221, 2006.

[40] CHIDIAC, S.E., et al., "Slump and slump flow for characterizing yield stress of fresh concrete", ACI materials journal, v. 103, n. 6, pp. 413, 2006.

[41] MURATA, J., KUKAWA, H., "Viscosity equation for fresh concrete”, Materials Journal, v. 89, n. 3, pp. 230-237, 1992.

[42] TANIGAWA, Y., et al., "Rheological study on slumping behavior of fresh concrete", Transactions of the Japan Concrete Institute, v. 14, pp. 1-8, 1992.

[43] HU C., Rhéologie des Bétons Fluides, Tese de D.Sc., ENPC. Études et Recherches des LPC, France, 1995.

[44] CHIDIAC, S.E., HABIBBEIGI, F., "Modelling the rheological behaviour of fresh concrete: An elastoviscoplastic finite element approach”, Computers and Concrete, v. 2, n. 2, pp. 97-110, 2005.

[45] TANIGAWA, Y., MORI, H., "Rheological Analysis of Slumping Behavior of Fresh Concrete”, In: Proceedings of the 29th Japan Congress on Materials Research, pp. 129-36, 1985.

[46] CHIDIAC, S.E., et al., "Controlling the quality of fresh concrete - a new approach", Magazine of Concrete Research, v. 52, n. 5, pp. 353-363, 2000.

[47] SCHOWALTER, W.R., CHRISTENSEN, G., "Toward a rationalization of the slump test for fresh concrete: comparisons of calculations and experiments", Journal of Rheology, v. 42, n. 4, pp. 865-870, 1998.

[48] CHIDIAC, S.E., MAHMOODZADEH, F., "Plastic viscosity of fresh concrete - A critical review of predictions methods", Cement and Concrete Composites, v. 31, n. 8, pp. 535-544, 2009.

[49] MAHMOODZADEH, F., CHIDIAC, S.E., "Rheological models for predicting plastic viscosity and yield stress of fresh concrete", Cement and Concrete Research, v. 49, pp. 1-9, 2013.

[50] CASTRO, A.L., LIBORIO, J.B.L., "Initial rheological description of high performance concretes", Materials Research, v. 9, n. 4, pp. 405-410, 2006.

\section{ORCID}

João Batista Pereira

Geraldo de Freitas Maciel
https://orcid.org/0000-0001-7136-917X

https://orcid.org/0000-0003-1272-9045 


\section{APÊNDICE A}

Tabela 4: Dados experimentais obtidos a partir do slump test padrão e equipamento automatizado; e parâmetros reológicos calculados a partir dos modelos de Ferraris e de Larrard (1998) e medidas obtidas no equipamento desenvolvido.

\begin{tabular}{|c|c|c|c|c|c|c|c|c|}
\hline \multirow[b]{2}{*}{ Ensaio } & \multirow[b]{2}{*}{ Traço } & \multirow[b]{2}{*}{$a / c$} & \multicolumn{2}{|c|}{ Slump (mm) } & \multirow{2}{*}{$\begin{array}{c}\text { Slump test padrão } \\
\text { Tensão limite de escoamento } \\
(\mathrm{Pa})^{\star *}\end{array}$} & \multicolumn{3}{|c|}{ Slump test automatizado } \\
\hline & & & padrão & automatizado & & $\begin{array}{l}\text { Tempo parcial de abatimento } \\
(\mathrm{s})^{*}\end{array}$ & $\begin{array}{l}\text { Tensão limite de escoamento } \\
(\mathrm{Pa})^{\star \star}\end{array}$ & $\begin{array}{c}\text { Viscosidade } \\
\text { plástica (Pa.s) * }\end{array}$ \\
\hline 1 & \multirow{8}{*}{$\mathrm{T} 1$} & \multirow{8}{*}{0,54} & 74,00 & 71,60 & - & - & - & - \\
\hline 2 & & & 73,70 & 68,20 & - & - & - & - \\
\hline 3 & & & 67,70 & 61,30 & - & - & - & - \\
\hline 4 & & & 64,00 & 60,60 & - & - & - & - \\
\hline 5 & & & 77,00 & 64,80 & - & - & - & - \\
\hline 6 & & & 78,70 & 62,60 & - & - & - & - \\
\hline 7 & & & 79,00 & 73,40 & - & - & - & - \\
\hline 8 & & & 76,00 & 67,70 & - & - & - & - \\
\hline 1 & \multirow{8}{*}{$\mathrm{T} 2$} & \multirow{8}{*}{0,56} & 119,00 & 114,80 & 1471,20 & 1,60 & 1500,40 & 96,60 \\
\hline 2 & & & 133,40 & 130,20 & 1371,00 & 2,05 & 1393,25 & 123,30 \\
\hline 3 & & & 120,70 & 120,00 & 1459,35 & 1,95 & 1464,20 & 117,30 \\
\hline 4 & & & 138,60 & 137,70 & 1334,823 & 1,35 & 1341,10 & 81,20 \\
\hline 5 & & & 143,30 & 139,50 & 1302,15 & 1,35 & 1328,55 & 81,20 \\
\hline 6 & & & 145,70 & 134,20 & 1285,45 & 1,30 & 1365,45 & 78,20 \\
\hline 7 & & & 121,70 & 111,00 & 1452,40 & 1,60 & 1526,85 & 96,60 \\
\hline 8 & & & 149,50 & 137,90 & 1259,00 & 1,25 & 1339,70 & 75,20 \\
\hline 1 & \multirow{8}{*}{$\mathrm{T} 3$} & \multirow{8}{*}{0,59} & 180,50 & 179,50 & 1047,45 & 1,15 & 1054,45 & 69,75 \\
\hline 2 & & & 184,30 & 176,70 & 1020,90 & 1,35 & 1074,05 & 81,90 \\
\hline 3 & & & 201,00 & 193,40 & 904,15 & 1,25 & 957,30 & 75,80 \\
\hline 4 & & & 188,80 & 186,60 & 989,45 & 1,25 & 1004,80 & 75,80 \\
\hline 5 & & & 195,30 & 188,80 & 944,00 & 1,15 & 989,45 & 69,75 \\
\hline 6 & & & 205,00 & 200,00 & 876,15 & 1,10 & 911,15 & 72,05 \\
\hline 7 & & & 206,70 & 202,20 & 864,30 & 1,00 & 895,75 & 71,25 \\
\hline 8 & & & 196,30 & 190,00 & 937,00 & 1,00 & 981,05 & 60,65 \\
\hline
\end{tabular}

* Para concretos com abatimento inferior a $100 \mathrm{~mm}$ não é possível medir o tempo parcial de abatimento (T) e o cálculo da viscosidade plástica; ** O modelo de previsão de tensão limite de escoamento vale para $\mathrm{S}>100 \mathrm{~mm}$. 